\title{
Tetrahydrobiopterin in Solid Organ Transplantation
}

\section{Manuel Maglione*}

Department of Visceral, Transplant and Thoracic Surgery, Innsbruck Medical University, Anichstrasse 35, A-6020 Innsbruck, Austria

Tetrahydrobiopterin is known to date as an essential cofactor of a set of 8 different enzymes including three nitric oxide synthase isoforms (neuronal, endothelial, and inducible), four tetrahydrobiopterin dependent aromatic amino hydroxylases (phenylalanine-, tyrosine-, and tryptophan hydroxylase 1 and 2), and the recently described alkylglycerol monooxygenase. Since the products of the tetrahydrobiopterin dependent reactions are crucially involved in a wide range of biological processes it is plausible that its $d e$ novo biosynthesis occurs in almost every cell of higher organisms [1].

While this compound is well known in cardiovascular research and in the field of metabolic disorders (tetrahydrobiopterin deficient hyperphenylalaninaemia) tetrahydrobiopterin is hardly present in transplantation literature.

In cardiovascular research several experimental studies, performed in animal models as well as in humans, revealed that the depletion of this essential co-factor in endothelial cells leads to endothelial dysfunction. The underlying mechanism is the "uncoupling" of the endothelial nitric oxide synthase. The term "uncoupling" refers to a dissociation of the electron flow occurring during the enzymatic process. As a consequence the endothelial nitric oxide synthase switches from a nitric oxide producing enzyme to an enzyme producing superoxide anions $\left(\mathrm{O}_{2}^{-}\right)$due to an increased reduction of oxygen. "Uncoupling" has been observed in diabetes, hyperlipidaemia and arterial hypertension, all cardiovascular pathologies associated with oxidative stress and endothelial dysfunction. Moreover, tetrahydrobiopterin substitution has been shown to prevent endothelial dysfunction in tissues subjected to ischemia reperfusion injury [2].

Ischemia reperfusion injury with its long term consequences represents a major challenge in solid organ transplantation, probably second only to organ shortage. Implementation of extended criteria donors and organs derived from donors after cardiac death demand further research in this field since these organs are definitely more vulnerable to this injury.

There are only a few research groups working on this co-factor in the field of solid organ transplantation, however, the results achieved are interesting. Of note, tetrahydrobiopterin has been shown to prevent not only ischemia reperfusion injury following solid organ transplantation, but there are also consisting experimental data showing immunosuppressive properties in acute rejection models. In experimental pancreas transplantation it has been nicely shown that tetrahydrobiopterin protects from ischemia reperfusion injury targeting the donors' nitric oxide synthases [3]. Based on cardiovascular research findings the proposed mechanism is the prevention of nitric oxide synthase "uncoupling", and therefore the prevention of endothelial dysfunction in the reperfused organ. However, the exact mechanism has not been described so far. Recent findings showed statins, another promising compound already popular in cardiovascular medicine, to protect transplanted murine hearts form ischemia reperfusion injury by increasing the activity of GTP-cyclohydrolase 1 [4]. GTPcyclohydrolase 1 is the key enzyme of tetrahydrobiopterin biosynthesis. Hence, the protective role of statins is another clue for the crucial role of tetrahydrobiopterin in ischemia reperfusion injury.

Different groups could demonstrate that tetrahydrobiopterin prevents acute rejection in experimental heart transplantation.
These findings were independently of the presence/activity of nitric oxide synthases [5,6]. It can be suggested that one of the other tetrahydrobiopterin dependent enzymes might be involved in acute rejection processes. Interestingly, the tetrahydrobiopterin dependent tryptophan hydroxylase has been recently shown to be crucially involved in tolerance induction [7]. This adds another exciting aspect to this compound.

Finally, own unpublished data suggest that tetrahydrobiopterin supplementation prevents transplant vasculopathy by suppressing endothelial activation. Again, the exact mechanism is not clear, however, it might be a consequence of diminishing ischemia reperfusion injury in the transplanted organ.

Current knowledge describes, therefore, Tetrahydrobiopterin as a compound which can prevent different pathologies in solid organ transplantation revealing novel, interesting targets in this research field. As with all experimental data, these results have to be interpreted with caution since rodents are not men. However, tetrahydrobiopterin has two big advantages: (a) the broad knowledge in cardiovascular research which can be in part translated into transplantation research, (b) the approvation by the FDA (United States Food and Drug Administration) and the EMEA (European Medicines Agency) for oral tetrahydrobiopterin administration in humans. Apart from a few studies where it was successfully administered to treat hypertension and hypercholesteremia, tetrahydrobiopterin is the therapy of choice for people suffering from tetrahydrobiopterindeficient hyperphenylalanineamia. The oral supplementation with the tetrahydrobiopterin precursor sapropterin dihydrochloride $\left(\right.$ Kuvan $^{\circledR}$, BioMarin, Tiburon, CA, USA) lowers phenylalanine levels in many patients independently of their dietary intake. So far no major adverse events were registered for oral tetrahydrobiopterin or Kuvan ${ }^{\mathbb{R}}$ administration [8] as well as in a recent human study for intravenous application of $20 \mathrm{mg} / \mathrm{kg}$ b.w. [9].

There are some caveats. A clinical trial showed that oral administration of $\operatorname{Kuvan}^{\circledR}$ had no effect on endothelial functionin patients with coronary artery disease. Systemic and vascular oxidation of tetrahydrobiopterin limits currently its oral application in cardiovascular diseases making new application strategies necessary [10]. In solid organ transplantation intravenous administration would be desirable since donor preconditioning is generally accepted to be the best strategy to prevent ischemia reperfusion injury. A stable formula for intravenous administration has still to be developed.

However, if familiar with the huge amount of experimental in vitro

${ }^{*}$ Corresponding author: Manuel Maglione, Center of Operative Medicine Department of Visceral, Transplant and Thoracic Surgery Innsbruck Medical University, Anichstrasse 35, A-6020 Innsbruck, Austria, E-mail: manuel.maglione@i-med.ac.at

Received August 29, 2013; Accepted August 29, 2013; Published August 31 2013

Citation: Maglione M (2013) Tetrahydrobiopterin in Solid Organ Transplantation. J Transplant Technol Res 3: e122. doi:10.4172/2161-0991.1000e122

Copyright: ( 2013 Maglione M. This is an open-access article distributed under the terms of the Creative Commons Attribution License, which permits unrestricted use, distribution, and reproduction in any medium, provided the original author and source are credited. 
and in vivo studies dealing with novel and promising treatments able to protect transplanted organs, a future clinical application of an already FDA/EMEA approved drug like tetrahydrobiopterin seems more likely compared to other compounds. Description of the mechanism of action in the prevention of ischemia reperfusion injury and acute rejection and, in parallel, development of a stable intravenous formula could be the first step to definitely path the way into clinical trials.

\section{References}

1. Werner ER, Blau N, Thöny B (2011) Tetrahydrobiopterin: biochemistry and pathophysiology. Biochem J 438: 397-414.

2. Katusic ZS, d'Uscio LV, Nath KA (2009) Vascular protection by tetrahydrobiopterin: progress and therapeutic prospects. Trends Pharmacol Sci 30: 48-54.

3. Maglione M, Cardini B, Oberhuber R, Watschinger K, Jenny M, et al. (2012) Prevention of lethal murine pancreas ischemia reperfusion injury is specific for tetrahydrobiopterin. Transpl Int 25: 1084-1095.

4. Tuuminen R, Syrjälä S, Krebs R, Keränen MA, Koli K, et al. (2011) Donor simvastatin treatment abolishes rat cardiac allograft ischemia/reperfusion injury and chronic rejection through microvascular protection. Circulation 124:11381150.

5. Brandacher G, Maglione M, Schneeberger S, Obrist P, Thoeni G, et al. (2006) Tetrahydrobiopterin compounds prolong allograft survival independently of their effect on nitric oxide synthase activity. Transplantation 81: 583-589.

6. Pieper GM, lonova IA, Cooley BC, Migrino RQ, Khanna AK, et al. (2009) Sepiapterin decreases acute rejection and apoptosis in cardiac transplants independently of changes in nitric oxide and inducible nitric-oxide synthase dimerization. J Pharmacol Exp Ther 329: 890-899.

7. Nowak EC, de Vries VC, Wasiuk A, Ahonen C, Bennett KA, et al. (2012) Tryptophan hydroxylase-1 regulates immune tolerance and inflammation. J Exp Med 209: 2127-2135.

8. Trefz FK, Scheible D, Frauendienst-Egger G, Korall H, Blau N (2005) Long-term treatment of patients with mild and classical phenylketonuria by tetrahydrobiopterin. Mol Genet Metab 86 Suppl 1: S75-80.

9. Artunc F, Essig M, Artunc N, Plachtzik C, Reich M, et al. (2008) Effects of tetrahydrobiopterin on nitric oxide bioavailability and renal hemodynamics in healthy volunteers. J Nephrol 21: 850-860.

10. Cunnington C, Van Assche T, Shirodaria C, Kylintireas I, Lindsay AC, et al. (2012) Systemic and vascular oxidation limits the efficacy of oral tetrahydrobiopterin treatment in patients with coronary artery disease. Circulation 125: 1356-1366. 